\title{
Biomasse et minéralomasse d'un taillis à courte révolution de peuplier Beaupré installé sur un sol acide hors vallée, amélioré par fertilisation
}

\author{
D Gelhaye, J Ranger*, M Bonneau \\ Équipe Cycles biogéochimiques, Centre Inra de Nancy, 54280 Champenoux
}

(Reçu le 25 juillet 1996: accepté le 18 novembre 1996)

\begin{abstract}
Summary - Biomass and nutrient content of a short rotation coppice of Beaupré poplars planted on a non-alluvial acidic soil improved by fertilization. Improvement by fertilization was tested on the production of a short rotation coppiced (SRC) stand of Beaupré poplar in LuthenayUxeloup forest (Nièvre-France). Several treatments bringing P, NPK or NPKCa were compared to a control without any improvement in a bloc experiment. The improvement of production was significant in all the treaments when compared to the control. NPKCa treatment was the best one. The absolute value of production is too low for an industrial development of short rotation forestry in these ecological conditions where water availability during the vegetation period seemed to be the primary limiting factor. The real potential of the site was not completly identified owing to the bad conditions for stand installation and the dry climate during the $1980 \mathrm{~s}$. Efficiency of nutrients for dry matter production in this short rotation coppice was more important than in the other stands previously investigated. Soil mineral enrichment was already apparent at the end of the first rotation. It will be necessary to test a fertilization restoring the nutrients drained during harvesting, on the production of the next generation.
\end{abstract}

\section{short rotation forestry / biomass / nutrient content / fertilization}

Résumé - Un essai d'amélioration par fertilisation minérale de la production d'un taillis à courte révolution (TCR) de peuplier Beaupré installé sur un sol forestier hors vallée, a été réalisé en forêt de Luthenay-Uxeloup (Nièvre). Divers traitements apportant $\mathrm{P}, \mathrm{NPK}$ ou NPKCa ont été comparés à un témoin sans fertilisation. La récolte du TCR après 9 années de végétation indique que l'effet de tous les traitements est significatif sur la production avec une supériorité du traitement apportant le calcium. La valeur absolue de la production moyenne annuelle du meilleur traitement reste très modeste $\left(<5 \mathrm{tha}^{-1} \cdot \mathrm{an}^{-1}\right)$ pour que cette culture ait un avenir réel pour la production de biomasse. Ces taillis installés dans de mauvaises conditions ont de plus subi les aléas climatiques des années 1980, de sorte que la production mesurée ne représente pas réellement le potentiel de la station. L'efficience de ce

\footnotetext{
* Correspondance et tirés à part

Tél : (33) 0383394068 ; fax : (33) 0383394069 ; courriel : ranger@nancy.inra.fr
} 
TCR dans l'utilisation des éléments minéraux pour produire la biomasse est importante et supérieure à celle des TCR habituellement étudiés. Les sols restent enrichis en éléments minéraux et une simple fertilisation de restitution des éléments exportés par la coupe devrait suffire pour une future génération dont il serait utile de mesurer la production.

taillis à courte révolution / biomasse / minéralomasse / fertilisation

\section{INTRODUCTION}

Les taillis à courte révolution (TCR) représentent une méthode efficace pour produire rapidement de la biomasse lignocellulosique. Le seuil de rentabilité de cette culture se situe au dessus d'une production d'une dizaine de tonnes de matière sèche aérienne totale par hectare et par an, pour une rotation comprise entre 7 et 10 ans, dont 75 à $80 \%$ sont utilisables pour la trituration (Afocel, 1982 ; Bonduelle et Berthelot, 1994a). Les surfaces affectées doivent permettre d'assurer un approvisionnement soutenu de produits calibrés pour une utilisation spécilique, assurant, comme toute production industrielle, l'approvisionnement des unités de traitement qu'il s'agisse de papeteries, d'unités quelconques de transformation, voire de centrales de combustion.

Les essais mis en place dans les années 1970 ont surtout concerné des implantations dans les conditions écologiques les plus favorables, autorisant une production élevée pendant la durée la plus courte possible. Les sites les plus favorables sont les sols alluviaux riches et potentiellement bien alimentés en eau pendant la saison de végétation. Les essences sont celles rejetint de souche assurant une régénération peu coûteuse et rapide : les genres Populus ont été les plus utilisés en France (Afocel, 1982 ; Bonduelle, 1989 ; Bonduelle et Berthelot, 1994a), les Suédois ont développé la culture du genre Salix (Perttu, 1984 ; Christersson et Sennerby-Forsse, 1995) et les Nord-Américains les Populus, Platanus et Liriodendron (Steinbeck et May, 1971 ; Carlisle et Methven, 1979; van Miegroet et al, 1994).
Compte tenu des terres potentiellement libérables par l'agriculture, ces cultures industrielles peuvent être amenées à se développer sur des sols plus pauvres, situés hors vallée où la production sera plus faible. II était donc intéressant d'acquérir de l'information sur ces plantations en conditions moins favorables, en testant l'intérêt de l'amélioration de la fertilité minérale du sol qui est un des facteurs limitants des TCR utilisant des essences améliorées (Shelton et al, 1982). Tel était l'objectif de l'essai d'introduction d'un TCR sur un ancien site forestier en forêt communale de LuthenayUxeloup (Nièvre).

Les objectifs de cet article sont les suivants :

- présenter les résultats de production de cette plantation de peuplier en fonction des fertilisations testées,

- quantifier le contenu minéral de tels peuplements de façon à prévoir leurs besoins nutritifs et les fertilisations optimales,

- comparer ces résultats à ceux obtenus dans des TCR de peupliers installés dans des conditions plus favorables.

\section{MATÉRIEL ET MÉTHODES}

L'essai de fertilisation de TCR de peuplier présenté dans cette étude est situé en forêt communale de Luthenay-Uxeloup (Nièvre). Il a été installé en 1984 sur une défriche de taillis-sousfutaie de chêne sessile (Quercus sessiliflora $\mathrm{L}$ ) et de charme (Carpinus betula L). Le site a été préparé par andainage des rémanents d'exploitation à l'aide d'un tracteur équipé d'une lame coupante, suivi d'un labour à la charrue à disques. Les conditions d'installation n ont pas été opti- 
Tableau I. Les traitements de fertilisation appliqués dans le dispositif.

\begin{tabular}{|c|c|c|c|c|c|c|c|c|c|}
\hline \multirow[t]{3}{*}{ Témoin } & \multirow{2}{*}{\multicolumn{3}{|c|}{$\frac{\text { En plein } k g . h a^{-1}}{\text { Mars } 1985}$}} & \multicolumn{4}{|c|}{ En g par plant } & \multicolumn{2}{|c|}{ En plein kg.ha' } \\
\hline & & & & \multirow{2}{*}{$\frac{\text { Déc } 85}{P_{2} O_{5}}$} & \multirow{2}{*}{$\frac{\text { Mai } 86}{\mathrm{~K}_{2} \mathrm{O}}$} & \multicolumn{2}{|c|}{ Mars 1987} & \multirow{2}{*}{$\frac{1990}{N}$} & \multirow{2}{*}{$\frac{1992}{N}$} \\
\hline & $\mathrm{P}_{2} \mathrm{O}_{5}$ & $\mathrm{~K}_{2} \mathrm{O}$ & $\mathrm{CaO}$ & & & $\mathrm{P}_{2} \mathrm{O}_{5}$ & $N$ & & \\
\hline $\mathrm{Pl}$ & 160 & & & 16 & & 20,2 & & & \\
\hline PlK1 & 160 & 160 & & 16 & 20 & 20,2 & & & \\
\hline $\mathrm{NP}_{1} \mathrm{~K}_{1}$ & 160 & 160 & & 16 & 20 & 20,2 & 8,4 & 150 & 150 \\
\hline $\mathrm{NP}_{2} \mathrm{~K}_{1}$ & 240 & 160 & & 32 & 20 & 40,4 & 8,4 & 150 & 150 \\
\hline $\mathrm{NP}_{1} \mathrm{~K}_{1} \mathrm{Ca}$ & 160 & 160 & 1120 & 16 & 20 & 20,2 & 8,4 & 150 & 150 \\
\hline
\end{tabular}

males avec un labour trop superficiel en période humide ne permettant pas un enfouissement suffisant des boutures.

Les conditions écologiques sont les suivantes : - le climat est caractérisé par une pluviométrie moyenne annuelle de $760 \mathrm{~mm}$ et une température moyenne annuelle de $10^{\circ} \mathrm{C}$. Les pluies sont distribuées de manière relativement homogène sur toute l'année ( 166 jours de pluie par an à Nevers-Fourchambault).

- les sols sont des sols lessivés à pseudogley développés sur des limons recouvrant des formations argileuses pliocènes d'origine continentale dites «sables et argiles du Bourbonnais». L'épaisseur des limons est d'environ 40 à $45 \mathrm{~cm}$.

Le peuplement de peuplier Beaupré (Populus trichocarpa $\times$ Populus deltoides) est issu de boutures mises en terre en mars 1984, à la densité de 3300 boutures par hectare, butées à l'automne 1984, et recépées en 1986 de façon à créer une situation de taillis. Un dépressage ne laissant qu'un seul brin par cépée a été réalisé en mars 1987 pour éviter la concurrence intra-cépée. La reprise des boutures en 1985 était de $93 \%$ dans le témoin, $85 \%$ dans le traitement $\mathrm{P}_{1}, 76 \%$ dans le traitement $\mathrm{NP}_{2} \mathrm{~K}_{1}$ et $77 \%$ dans le traitement $\mathrm{NP}_{1} \mathrm{~K}_{1} \mathrm{Ca}$

Les traitements de fertilisation appliqués à la surface du sol sans enfouissement, sont présentés dans le tableau I. Un desherbage manuel a été effectué autour des boutures pendant 2 ans ainsi qu'un traitement mécanique au «rotovator» entre les lignes.

Compte tenu des résultats obtenus, les mesures de biomasse et minéralomasse ont porté sur les traitements les plus intéressants: $\mathrm{P}_{1}$, $\mathrm{NP}_{2} \mathrm{~K}_{1}, \mathrm{NP}_{1} \mathrm{~K}_{1} \mathrm{Ca}$ qui seront comparés au témoin (T) n' ayant reçu aucune fertilisation.
Les résultats présentés concernent des taillis dont l'ensouchement a 11 ans et les rejets 9 ans.

\section{Échantillonnage}

- Les sols : un profil pédologique a été décrit par bloc dans chacun des traitements retenus ( 16 fosses principales). L'échantillonnage a porté sur ce profil principal et sur deux sondages «satellites» réalisés à la tarière pédologique, de façon à intégrer la variabilité spatiale intra-placette. Les trois échantillons recueillis par horizon ont été mélangés lors du prélèvement pour ne conserver qu'un profil moyen par traitement et par bloc, soit 16 profils moyens.

-Les peuplements : 15 tiges ont été sélectionnées dans chaque traitement à partir de l'inventaire de chaque placette. Ces arbres sont distribués sur toutes les classes de diamètre. Les 15 arbres sont répartis dans chacun des blocs en faisant l'hypothèse que l'effet bloc ne se traduit pas au niveau des relations biométriques (tarifs), hypothèse qu"il est possible de tester sur un nombre toutefois réduit d'individus.

Pour chaque arbre les mesures suivantes ont été effectuées : hauteur totale et hauteur jusqu'à la découpe $4 \mathrm{~cm}$ (D4), la circonférence de la souche jusqu'au fin-bout de la tige, tous les deux mètres et la circonférence à $1,30 \mathrm{~m}\left(\mathrm{C}_{130}\right)$.

Cess arbres ont été débités en compartiments (tronc à la découpe $4 \mathrm{~cm}$ et fin bout, branches vivantes, branches mortes). Ces compartiments ont été pesés globalement sur le terrain et des échantillons représentatifs ont été prélevés pour la mesure des taux d'humidité (à $65^{\circ} \mathrm{C}$ jusqu'à poids constant) et les analyses chimiques. Les prélèvements ont eu lieu en avril 1995, au début de la phase de débourrement. 
L'ensemble de ces résultats permettent :

i) la mesure de la matière sèche et du contenu minéral de chaque compartiment de chaque arbre;

ii) la mesure de la concentration en éléments minéraux majeurs ( 320 analyses) et l'étude de la distribution qualitative en fonction des compartiments et des traitements;

iii) la quantification de la minéralomasse compartimentée pour les différents compartiments des arbres échantillons;

iv) l'établissement de tarif's reliant sur cet échantillon, la biomasse ou la minéralomasse à un paramètre dendrométrique simple $\left(\mathrm{C}_{130}\right)$;

v) l'évaluation de la biomasse et de la minéralomasse du peuplement sur une base d'un hectare à partir de l'inventaire des placeaux et des tarifs.

\section{Les méthodes analytiques utilisées}

- Les sols : la granulométrie a été déterminée par la méthode Internationale; le carbone organique par la méthode Anne (1945); l'azote organique total par la méthode Kjeldhal ; le $\mathrm{pH}$ a été mesuré dans l'eau et dans une solution de $\mathrm{KCl} 1 \mathrm{~N}$ avec un rapport pondéral sol/solution de $1 / 2,5$; la capacité d'échange cationique et sa garniture ionique ont été déterminées par la méthode de Rouiller et al (1980) utilisant l'extraction par un sel non tamponné $(\mathrm{KCl} I \mathrm{~N})$ sauf pour $\mathrm{K}\left(\mathrm{NH}_{4} \mathrm{Cl}\right.$ $1 \mathrm{~N}$ ), le dosage des cations est réalisé par spectrométrie d'émission à plasma induit (Jobin Yvon JY $38+$ ) sauf pour $\mathrm{Al}$ et $\boldsymbol{H}$ déterminés par titrimétrie ; le phosphore assimilable a été déterminé par la méthode Duchaufour et Bonneau (1959); l'aluminium libre a été extrait par la méthode Deb à l'obscurité (Deb, 1960) et le fer libre par la méthode de Mehra et Jackson (1962).

- Le contenu minéral des végétaux a été déterminé après minéralisation par $\mathrm{H}_{2} \mathrm{O}_{2}$ et $\mathrm{HClO}_{4}$ à chaud et dosage des cations et du phosphore par spectrométrie d'émission à plasma induit (Jobin Yvon JY $38^{+}$) ; l'azote est dosé après minéralisation spécifique en milieu sulfurique en présence d'un catalyseur et dosage colorimétrique sur autoanalyseur Technicon II.

\section{RÉSULTATS}

\section{Les sols}

Ces sols polygéniques se caractérisent par une rupture granulométrique entre des hori- zons de surface présentant un taux d'argile inférieur à $15 \%$ et des horizons inférieurs dont le taux d'argile est supérieur à $40 \%$; ces deux groupes d'horizons sont séparés par un horizon intermédiaire de mélange des deux matériaux et/ou de dégradation hydromorphe. La densité apparente est élevée sur tout le profil de sol et supérieure à 1,2 en surface voire 1,5 entre 15 et $30 \mathrm{~cm}$ de profondeur. L'enracinement est concentré dans les 45 premiers $\mathrm{cm}$ de sol. $\mathrm{Le} \mathrm{pH}_{\text {eau }}$ n'est que modérément acide $(4,6<\mathrm{pH}<5,3)$ mais le $\mathrm{pH}_{\mathrm{KCl}}$ nettement plus faible traduit une forte acidité d'échange et une désaturation notable. Le profil de fer libre indique bien la présence d'un horizon de pseudogley dans lequel cet élément est remobilisé (tableau II).

Au plan agronomique, ces sols sont caractérisés par une rupture granulométrique forte vers $40-45 \mathrm{~cm}$ de profondeur, une structure faiblement à modérément exprimée, aussi bien dans les horizons superficiels limoneux que dans les horizons profonds argileux. Ils ont tendance à être pauvres en surface en $\mathrm{Ca}, \mathrm{Mg}$ et $\mathrm{P}$ mais plutôt bien pourvus en profondeur, sauf en $P$. L'effet des traitements de fertilisation est encore très apparent en particulier sur les teneurs en $\mathrm{P}$ et $\mathrm{Ca}$ dans les traitements apportant ces éléments. L'apport d'azote semble encore apparaître directement ou plus vraisemblablement indirectement, par des restitutions plus fortes dans les traitements fertilisés où le $\mathrm{C} / \mathrm{N}$ reste cependant très élevé. L'effet des traitements est d'ailleurs statistiquement significatif pour ce qui concerne le $\mathrm{pH}$, le taux de saturation et le calcium échangeable si on considère les 30 premiers $\mathrm{cm}$ de sol (NPIK $1 \mathrm{Ca}$ est différent des autres traitements); l'effet azote n'est pas significatif et celui de $P$ ne peut être testé faute d'un échantillonnage trop limité pour cet élément. Quand on considère le profil complet, les différences significatives ont trait au $\mathrm{pH}$ et au taux de saturation discriminant le témoin des traitements. L'analyse d'un profil granulomé- 


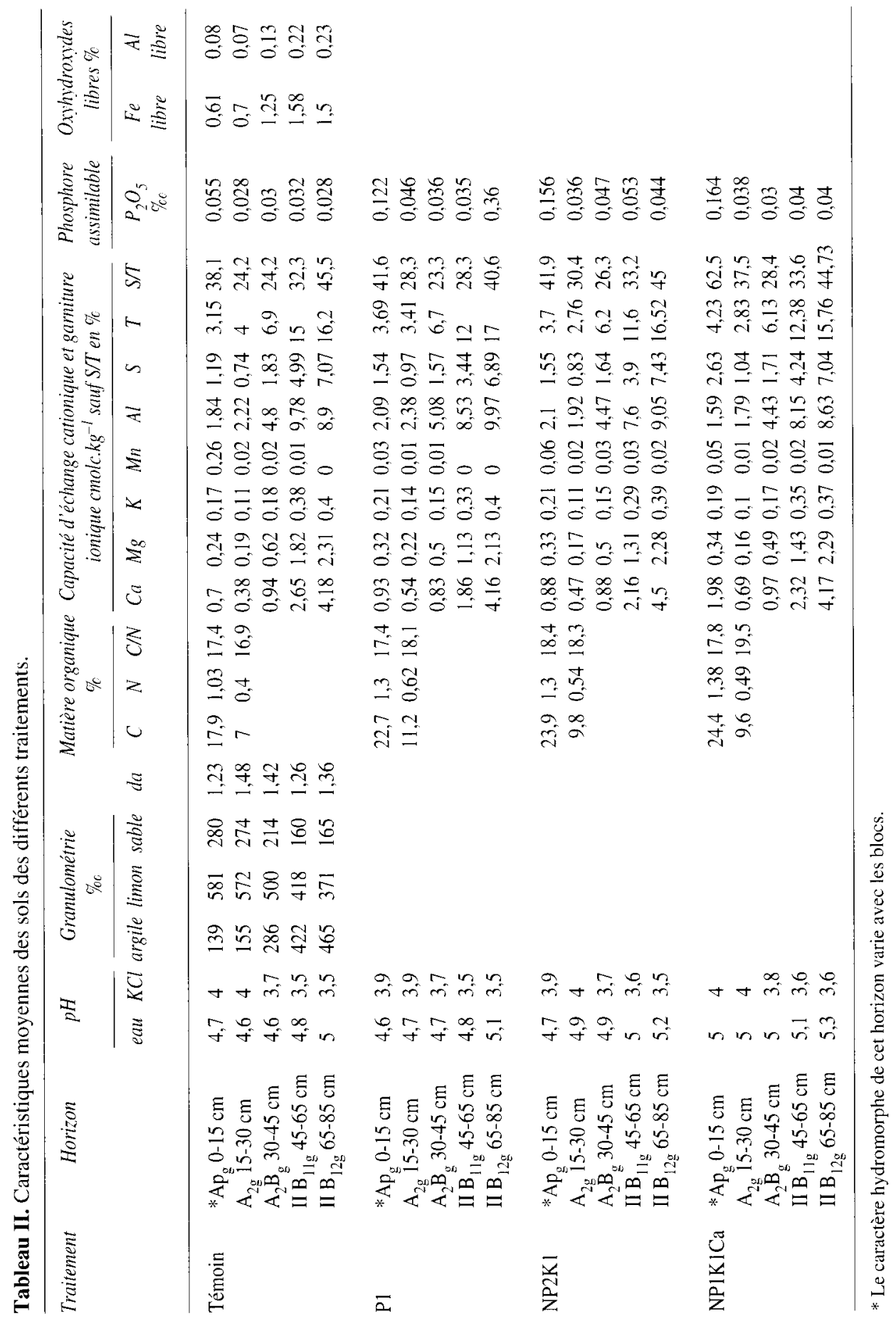


Tableau III. Masse d'éléments par hectare calculées pour deux profondeurs de sol (données en kilo par hectare).

\begin{tabular}{llcrrrrr}
\hline & & $C$ & $N$ & $C a$ & $M g$ & $K$ & $P_{2} O_{5}$ \\
\hline Témoin & Bilan sur 30 cm & 48566 & 2788 & 427 & 173 & 112 & 33 \\
& Bilan sur 85 cm & & & 4437 & 2611 & 597 & 77 \\
\multirow{2}{*}{ PI } & Bilan sur 30 cm & 66746 & 3775 & 583 & 216 & 140 & 65 \\
& Bilan sur 85 cm & & & 4137 & 2157 & 587 & 118 \\
\multirow{2}{*}{ NP2K1 } & Bilan sur 30 cm & 65852 & 3597 & 533 & 197 & 126 & 74 \\
& Bilan sur 85 cm & & & 4445 & 2311 & 549 & 144 \\
\multirow{2}{*}{ NP1K1Ca } & Bilan sur 30 cm & 66330 & 3634 & 1037 & 197 & 115 & 77 \\
& Bilan sur 85 cm & & & 4888 & 2372 & 565 & 132 \\
\hline
\end{tabular}

$\mathrm{C}$ et $\mathrm{N}$ totaux : $\mathrm{Ca}, \mathrm{Mg}$ et $\mathrm{K}$ échangeables; $\mathrm{P}_{2} \mathrm{O}_{5}$ assimilable (méthode Duchaufour-Bonneau, 1959).

trique par bloc (dans les traitements témoins) ne révèle aucune différence significative.

Les calculs quantitatifs de réserve des sols en éléments assimilables indiquent des sols moyennement pourvus où les apports de fertilisants restent quantifiables pour $\mathrm{P}$, $\mathrm{Ca}$; l'apport de K n'est pas apparent et celui de $\mathrm{N}$ n'apparaît vraisemblablement que par le jeu d'un cycle biologique plus actif dans les traitements fertilisés (tableau III).

L'effet bloc testé sur la granulométrie réalisée seulement dans les traitements témoins n'indique aucune différence entre blocs pour ce critère, mais l'échantillonnage est limité. Ce même effet testé sur les variables quantitatives et pour deux profondeurs de sol montre que :
- les réserves sur $30 \mathrm{~cm}$ de sol en $\mathrm{C}, \mathrm{N}$, $\mathrm{Ca}_{\text {ech }}, \mathrm{Mg}_{\text {ech }}$ et $\mathrm{K}_{\text {ech }}$ ne sont différentes que pour $\mathrm{Mg}$; le bloc 1 est plus pauvre que les autres blocs,

- les réserves sur $85 \mathrm{~cm}$ de profondeur ne sont différentes que pour $\mathrm{K}$; le bloc 1 s'isolant à nouveau des autres blocs, avec des réserves plus faibles.

\section{La croissance des peuplements}

Les mesures effectuées tous les ans depuis 1987 conduisent aux conclusions suivantes :

- $\mathrm{P}$ a eu rapidement un effet positif significatif sur la croissance en hauteur (tous les traitements différent du témoin) ; Ca a éga-

Tableau IV. Variabilité inter-blocs pour les inventaires concernant la circonférence à $1,30 \mathrm{~m}$ des brins dans les différents traitements (mesures de l'automne 1994 ; données en cm).

\begin{tabular}{lcccccc}
\hline & Bloc 1 & Bloc 2 & Bloc 3 & Bloc 4 & $\begin{array}{c}\text { Moyenne par } \\
\text { traitement }\end{array}$ & $\begin{array}{c}\text { test } \\
\text { Newman-Keuls* }\end{array}$ \\
\hline Témoin & 78 & 175 & 178 & 178 & 152 & $\mathrm{a}$ \\
$\mathrm{P}_{1}$ & 167 & 185 & 262 & 261 & 219 & $\mathrm{~b}$ \\
$\mathrm{NP}_{2} \mathrm{~K}_{1}$ & 278 & 314 & 291 & 322 & 301 & $\mathrm{c}$ \\
$\mathrm{NP}_{1} \mathrm{~K}_{1} \mathrm{Ca}$ & 259 & 349 & 360 & 284 & 313 & $\mathrm{c}$ \\
\hline
\end{tabular}

* Une lettre commune signifie que les traitements ne sont pas significativement différents au seuil de $5 \%$. 
lement un effet positif remarquable $\left(\mathrm{NP}_{1} \mathrm{~K}_{1} \mathrm{Ca}\right.$ significativement différent de $\mathrm{NP}_{1} \mathrm{~K}_{1}$ ).

- $\mathrm{P}$ et $\mathrm{K}$ ont eu un effet continu sur la croissance en circonférence avec un effet dose significatif pour chacun de ces éléments. Les effets de $\mathrm{Ca}$ et $\mathrm{N}$ disparaissent après 1993 ,

- une hétérogénéité importante existe dans cet essai (effet bloc significatif).

Les résultats des mesures de circonférence effectuées en 1994 figurent au tableau IV (Bonneau, 1994 ; 1995). Ils confirment l'effet bloc, qui agit en interaction avec les traitements (le bloc I comparé à la moyenne des blocs donne $-50 \%$ pour $\mathrm{T},-2,4 \%$ pour $\mathrm{P} 1,-7,6 \%$ pour $\mathrm{NP}_{2} \mathrm{~K}_{1}$ et $-17 \%$ pour $\mathrm{NP}_{1} \mathrm{~K}_{1} \mathrm{Ca}$ ), à moins que le bloc lui même ne soit hétérogène.

L'effet des traitements est important, avec un doublement de la circonférence moyenne, significatif pour les traitements $\mathrm{NP}_{2} \mathrm{~K}_{1}$ et $\mathrm{NP}_{1} \mathrm{~K}_{1} \mathrm{Ca}$; l'effet de Ca n'est plus signifi- catif par rapport à $\mathrm{NP}_{1} \mathrm{~K}_{1}$ et difficile à tester dans cet essai par rapport à $\mathrm{NP}_{2} \mathrm{~K}_{1}$ où l'apport de $\mathrm{P}$ a été le double de celui de $\mathrm{NP}_{1} \mathrm{~K}_{1} \mathrm{Ca}$.

L'effet sur la croissance en hauteur a également été important ; a titre d'exemple, la hauteur totale des brins était respectivement de 77, 71, 75 et $85 \mathrm{~cm}$ pour les traitements $\mathrm{T}, \mathrm{P} 1, \mathrm{NP}_{2} \mathrm{~K}_{1}$ et $\mathrm{NP}_{1} \mathrm{~K}_{1} \mathrm{Ca}$ en 1986 et 233 , 266, 298 et $407 \mathrm{~cm}$ en 1988.

\section{La production de matière sèche}

Les relations entre la biomasse d'un compartiment et la dimension des arbres sont indépendantes du traitement comme le montre l'exemple de la figure 1 . La forme des arbres, la branchaison ou la densité du bois ne sont pas affectées de manière significative, à cette échelle d'observation, par les traitements de fertilisation. Au vu de ces résultats, des tarifs communs à tous les traitements, reliant biomasse et circonférence

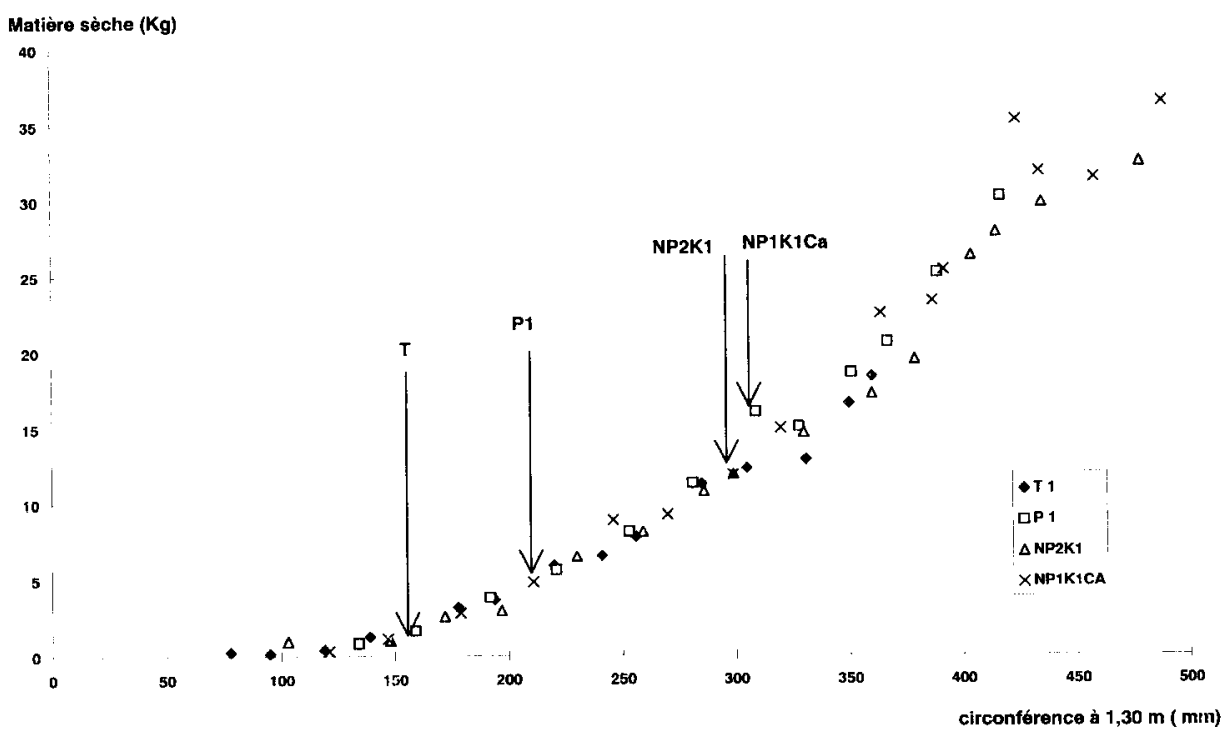

Fig 1. Relation biomasse-circonférence à $1,30 \mathrm{~m}$. La flèche indique la valeur de la circonférence moyenne pour chaque traitement. 
Tableau V. Les tarifs de biomasse pour les principaux compartiments.

\begin{tabular}{lll}
\hline Compartiment & $R^{2}$ \\
\hline $\begin{array}{l}\text { Souche/D4 } \\
\quad \text { Bois } \\
\text { Écorce }\end{array}$ & $\mathrm{Y}=0,000203 \mathrm{X}^{2}-0,019150 \mathrm{X}$ & 0,976 \\
Arbre total & $\mathrm{Y}=0,000026 \mathrm{X}^{2}-0,000747 \mathrm{X}$ & 0,981 \\
$\quad$ & \\
$\quad$ Bois & $\mathrm{Y}=0,000190 \mathrm{X}^{2}-0,012733 \mathrm{X}$ & 0,980 \\
$\quad$ & $\mathrm{Y}=0,000023 \mathrm{X}^{2}-0,000935 \mathrm{X}$ & 0,986 \\
Branches vivantes & $\mathrm{Y}=0,000062 \mathrm{X}^{2}-0,008390 \mathrm{X}$ & 0,875 \\
\hline
\end{tabular}

$\mathrm{Y}=$ biomasse du compartiment $; \mathrm{X}=$ circonférence à $1,30 \mathrm{~m}\left(\mathrm{C}_{130}\right)$.

Tableau VI. Variation de la biomasse compartimentée en fonction des blocs et des traitements (données en kilo de matière sèche par hectare).

\begin{tabular}{|c|c|c|c|c|c|}
\hline & $\begin{array}{c}\text { Bois } \\
\text { de tronc }\end{array}$ & $\begin{array}{l}\text { Ecorce } \\
\text { de tronc }\end{array}$ & $\begin{array}{l}\text { Branches } \\
\text { vivantes }\end{array}$ & $\begin{array}{c}\text { Branches } \\
\text { mortes }\end{array}$ & $\begin{array}{c}\text { M.S ligneuse } \\
\text { totale }\end{array}$ \\
\hline \multicolumn{6}{|l|}{ Témoin } \\
\hline Moyenne des blocs & 6575 & 1512 & 994 & 744 & $9825 a^{*}$ \\
\hline Coefficient de variation $(\%)$ & 64 & 57 & 71 & 59 & 63 \\
\hline \multicolumn{6}{|l|}{ P1 } \\
\hline Moyenne des blocs & 13895 & 2692 & 2789 & 1392 & $20768 \mathrm{ab}$ \\
\hline Coefficient de variation (\%) & 55 & 45 & 70 & 48 & 55 \\
\hline \multicolumn{6}{|l|}{ NP2K I } \\
\hline Moyenne des blocs & 22057 & 3832 & 5111 & 2048 & 33047 bc \\
\hline Coefficient de variation $(\%)$ & 24 & 26 & 22 & 25 & 24 \\
\hline \multicolumn{6}{|l|}{$\mathrm{NP} 1 \mathrm{~K} 1 \mathrm{Ca}$} \\
\hline Moyenne des blocs & 28586 & 4862 & 6808 & 2616 & $42873 \mathrm{c}$ \\
\hline Coefficient de variation $(\%)$ & 25 & 23 & 29 & 23 & 25 \\
\hline
\end{tabular}

* Des lettres différentes indiquent une différence entre traitements significative au seuil de $5 \%$ (test de NewmanKeuls).

à $1,30 \mathrm{~m}(\mathrm{C} 130)$, ont été établis. Les principaux tarifs sont présentés dans le tableau V.

L'effet des traitements sur la production de biomasse est important (tableau VI) : + $110 \%$ si on compare $P_{1}$ à $\mathrm{T},+236 \%$ si on compare $\mathrm{NP}_{2} \mathrm{~K}_{1}$ à $\mathrm{T}$ et $336 \%$ si on compare $\mathrm{NP}_{1} \mathrm{~K}_{1} \mathrm{Ca}$ à $\mathrm{T}$ pour la production de biomasse ligneuse aérienne totale. Les effets sont plus marqués en valeur relative pour la biomasse que pour les circonférences.
L'effet bloc est particulièrement important pour le traitement témoin où le bloc 1 a une production de l'ordre de $10 \%$ de la moyenne des blocs.

\section{Le contenu en éléments nutritifs des peuplements}

L'observation des variations de concentration en éléments nutritifs en fonction des traitements et compartiments montre une 
Tableau VII. Concentration moyenne en éléments majeurs par compartiment et traitement (données en \% de matière sèche à $65^{\circ} \mathrm{C}$ ).

\begin{tabular}{|c|c|c|c|c|c|c|c|c|}
\hline & \multicolumn{4}{|c|}{ Tronc } & \multicolumn{3}{|c|}{ Branches } & \multirow{3}{*}{$\begin{array}{c}\text { test } \\
\text { Newman- } \\
\text { Keuls }\end{array}$} \\
\hline & \multicolumn{2}{|c|}{ bois } & \multicolumn{2}{|c|}{ écorce } & \multirow[t]{2}{*}{ vivantes } & \multirow[t]{2}{*}{ mortes } & \multirow[t]{2}{*}{ feuilles } & \\
\hline & $\begin{array}{c}\text { souchel } \\
\text { D4 }\end{array}$ & $\begin{array}{c}\text { D4/fin } \\
\text { bout }\end{array}$ & $\begin{array}{c}\text { souchel } \\
\text { D4 }\end{array}$ & $\begin{array}{c}\text { D4/fin } \\
\text { bout }\end{array}$ & & & & \\
\hline \multicolumn{9}{|l|}{$\mathbf{N}$} \\
\hline Témoin & 0,15 & 0,13 & 0,41 & 0,79 & 0,47 & 0,20 & 3,64 & a \\
\hline P1 & 0,08 & 0,12 & 0,32 & 0,73 & 0,36 & 0,17 & 3,71 & a \\
\hline NP2K1 & 0,08 & 0,13 & 0,47 & 0,83 & 0,35 & 0,21 & 3,77 & a \\
\hline $\begin{array}{l}\text { NP1K1Ca } \\
* \text { test de }\end{array}$ & 0,15 & 0,15 & 0,49 & 0,96 & 0,41 & 0,19 & 3,98 & a \\
\hline Pewman-Keuls & $\mathrm{a}$ & a & b & $\mathrm{c}$ & b & & d & \\
\hline \multicolumn{9}{|l|}{$\mathbf{P}$} \\
\hline $\mathrm{Pl}$ & 0,01 & 0,02 & 0,06 & $\begin{array}{c}0,05 \\
0,1\end{array}$ & 0,05 & 0,0 & $\begin{array}{l}0,62 \\
0,66\end{array}$ & $\begin{array}{l}\mathrm{a} \\
\mathrm{a}\end{array}$ \\
\hline NP2K1 & 0,01 & 0,01 & 0,06 & 0,09 & 0,04 & 0,01 & 0,60 & a \\
\hline $\begin{array}{l}\text { NPlK } 1 \mathrm{Ca} \\
\text { test de }\end{array}$ & 0,01 & 0,01 & 0,05 & 0,08 & 0,04 & 0,01 & 0,59 & a \\
\hline Newman-Keuls & a & a & b & $\mathrm{c}$ & $\mathrm{b}$ & & $\mathrm{d}$ & \\
\hline \multicolumn{9}{|l|}{$\mathbf{K}$} \\
\hline Témoin & 0,08 & 0,09 & 0,5 & 0,64 & 0,33 & 0,07 & 1,69 & $\mathrm{a}$ \\
\hline P1 & 0,08 & 0,09 & 0,46 & 0,73 & 0,32 & 0,011 & 1,56 & a \\
\hline NP2K1 & 0,08 & 0,09 & 0,47 & 0,75 & 0,31 & 0,08 & 1,87 & a \\
\hline $\begin{array}{l}\text { NP1K1Ca } \\
\text { test de }\end{array}$ & 0,10 & 0,10 & 0,5 & 0,79 & 0,36 & 0,10 & 1,64 & a \\
\hline Newman-Keuls & a & a & $\mathrm{c}$ & $\mathrm{d}$ & $\mathrm{b}$ & & e & \\
\hline \multicolumn{9}{|l|}{ Ca } \\
\hline Témoin & 0,13 & 0,09 & 0,83 & 0,78 & 0,33 & 0,26 & 0,61 & a \\
\hline P1 & 0,10 & 0,10 & 0,78 & 0,82 & 0,34 & 0,24 & 0,82 & a \\
\hline NP2K1 & 0,09 & 0,09 & 0,83 & 0,80 & 0,28 & 0,27 & 0,71 & $\mathrm{a}$ \\
\hline $\begin{array}{l}\text { NP1K } 1 \mathrm{Ca} \\
\text { test de }\end{array}$ & 0,10 & 0,09 & 1,00 & 0,96 & 0,39 & 0,37 & 0,83 & a \\
\hline Newman-Keuls & a & a & d & d & $b$ & & $\mathrm{c}$ & \\
\hline \multicolumn{9}{|l|}{ Mg } \\
\hline Témoin & 0,05 & 0,04 & 0,21 & 0,23 & 0,11 & 0,05 & 0,41 & a \\
\hline P1 & 0,04 & 0,04 & 0,18 & 0,24 & 0,11 & 0,04 & 0,49 & a \\
\hline NP2K1 & 0,03 & 0,04 & 0,16 & 0,23 & 0,08 & 0,056 & 0,49 & a \\
\hline $\begin{array}{l}\text { NP1K1Ca } \\
\text { test de }\end{array}$ & 0,03 & 0,03 & 0,14 & 0,21 & 0,08 & 0,04 & 0,39 & a \\
\hline Newman-Keuls & a & a & $\mathrm{c}$ & d & b & & $\mathrm{e}$ & \\
\hline \multicolumn{9}{|l|}{$\mathbf{S}$} \\
\hline Témoin & 0,02 & 0,02 & 0,07 & 0,10 & 0,05 & 0,03 & 0,37 & $\mathrm{a}$ \\
\hline P1 & 0,02 & 0,02 & 0,07 & 0,09 & 0,05 & 0,02 & 0,36 & a \\
\hline NP2K1 & 0,02 & 0,02 & 0,07 & 0,010 & 0,04 & 0,03 & 0,35 & $\mathrm{a}$ \\
\hline $\begin{array}{l}\text { NP1K ICa } \\
\text { test de }\end{array}$ & 0,01 & 0,02 & 0,07 & 0,10 & 0,05 & 0,03 & 0,36 & a \\
\hline Newman-Keuls & a & a & $\mathrm{c}$ & $\mathrm{d}$ & b & & $\mathrm{e}$ & \\
\hline
\end{tabular}

* Une lettre différente indique une différence significative au seuil de $5 \%$ (test de comparaison de moyenne de Newman-Keuls). 
répartition classique : bois < écorce < feuilles (les compartiments mixtes, comme les branches ont une composition intermédiaire). Les résultats du tableau VII montrent que ces différences entre compartiments sont statistiquement significatives mais que l'effet des traitements ne l'est jamais sur la composition chimique à cette échelle d'observation. La forte concentration des feuilles est liée à leur stade végétatif (phase de débourrement) et ne peut être interprétée ici en termes de diagnostic foliaire classique. Les analyses foliaires de
1991 indiquaient une déficience nette en P et Ca du témoin non traité et une amélioration substantielle de la nutrition des peuplements, en particulier dans les traitements les plus complets $\left(\mathrm{NP}_{2} \mathrm{~K}_{1}\right.$ et $\left.\mathrm{NP}_{1} \mathrm{~K}_{1} \mathrm{Ca}\right)$ pour lesquels $\mathrm{N}, \mathrm{K}, \mathrm{Mg}$ et $\mathrm{Ca}$ semblent se situer à l'optimum, tandis que $\mathrm{P}$ se situe toujours en deçà (tableau VIII).

Ces résultats permettent de construire des tarifs de minéralomasse communs aux différents traitements pour un même compartiment (tableau IX). La figure 2 montre que

Tableau VIII. Résultats des analyses folaires effectuées en août 1991 (données en \% de matière sèche à $65^{\circ} \mathrm{C}$ ).

\begin{tabular}{lccccc}
\hline & $N$ & $P$ & $K$ & $C a$ & $M g$ \\
\hline Témoin & 2,04 & 0,09 & 1,63 & 0,37 & 0,31 \\
P1 & 1,87 & 0,14 & 1,63 & 0,49 & 0,35 \\
NP2K1 & 2,31 & 0,14 & 1,74 & 0,43 & 0,32 \\
NP1K 1Ca & 2,10 & 0,13 & 1,57 & 0,79 & 0,30 \\
Optimum norme Bonneau, 1995 & $2,0-2,5$ & $0,15-0,20$ & $1,5-1,8$ & $0,5-0,8$ & $>0,2$ \\
\hline
\end{tabular}

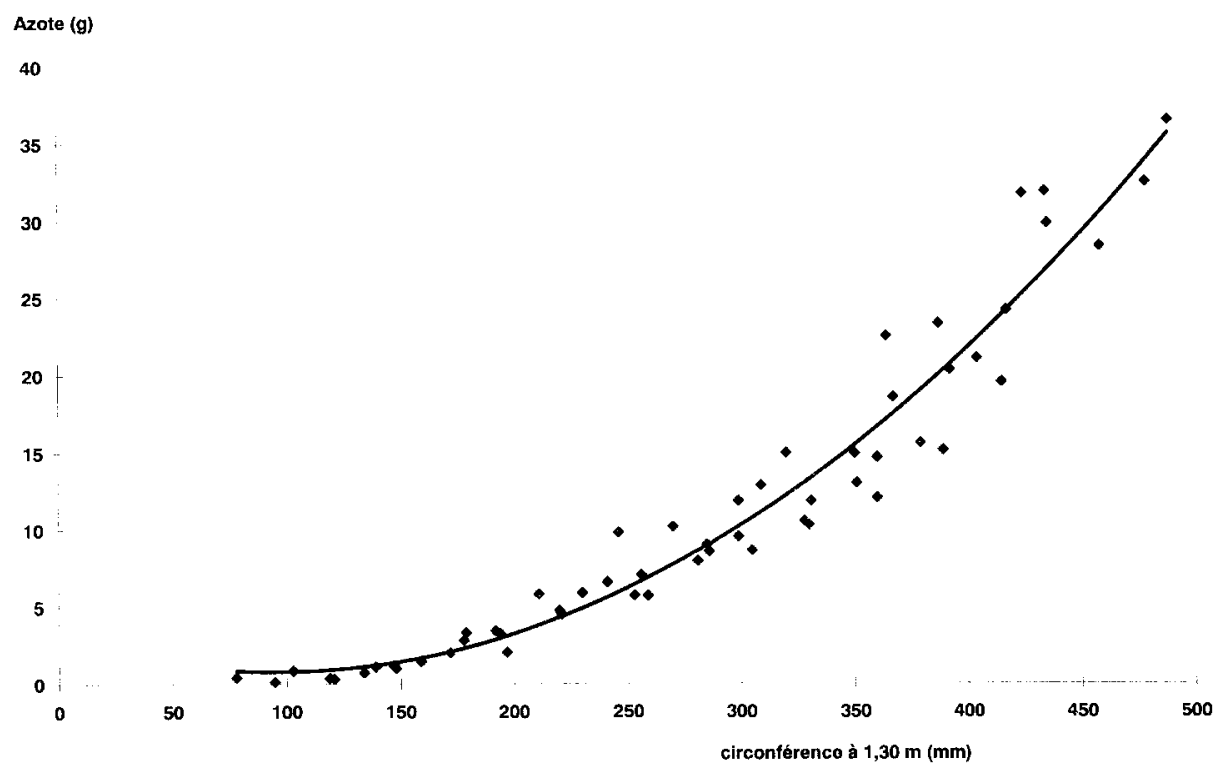

Fig 2. Relation minéralomasse-circonférence à $1,30 \mathrm{~m}$. 


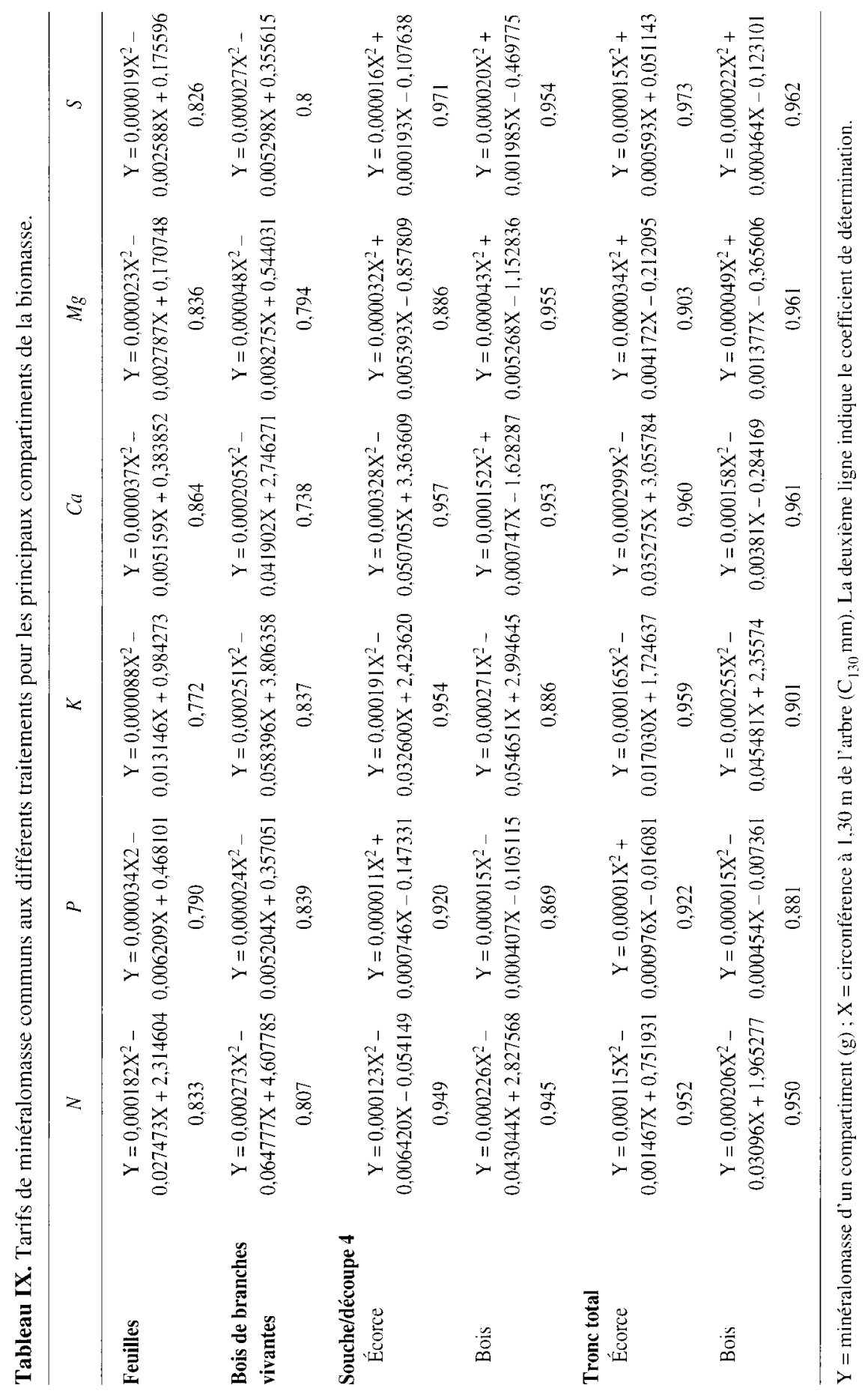




\begin{tabular}{|c|c|c|c|c|c|c|c|c|c|c|}
\hline \multirow{6}{*}{$\frac{\Xi}{\vdots}$} & $\infty$ & 寽空宫 & $\ddot{\theta}=$ & $\hat{\sigma}_{i} \underset{\sim}{8}$ & 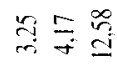 & \multirow{6}{*}{$\frac{\bar{s}}{\frac{\Xi}{2}}$} & $\infty$ & ఠ్ & $\hat{\sigma}$ & @of \\
\hline & $\sum$ & $\underset{\sim}{g} \underset{m}{0}$ & $\begin{array}{l}\infty \\
\stackrel{\infty}{\sigma} \\
=\end{array}$ & 哥志 & $\begin{array}{c}\approx \\
\infty\end{array}$ & & $\stackrel{\infty}{\Sigma}$ & b & $\ddot{8}$ & $\underset{g}{g} \stackrel{0}{g}$ \\
\hline & $\tilde{\Theta}$ & 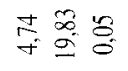 & 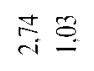 & 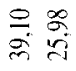 & 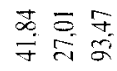 & & $\Xi$ & తి & 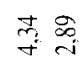 & $\begin{array}{l}88 \\
38 \\
+\infty\end{array}$ \\
\hline & 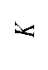 & $\begin{array}{l}8 \\
\varrho\end{array}$ & $\underset{i}{\infty}$ & $\begin{array}{ll}\infty & 0 \\
\infty & 0 \\
-1 & -1\end{array}$ & 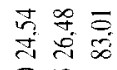 & & $\infty$ & $\stackrel{5}{s}$ & 等总 & 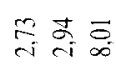 \\
\hline & $=$ & E 굥 & $\frac{2}{6} \frac{1}{6}$ & $\stackrel{\mathscr{S}}{\vec{i}} \vec{i}$ & 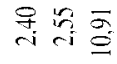 & & $=$ & $\ddot{2}$ & $\tilde{\sigma}$ & 동 \\
\hline & $z$ & 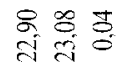 & $\underset{\infty}{\infty}$ & 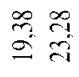 & 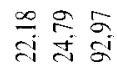 & & $z$ & in & $\underset{n}{\sim} \underset{r}{\stackrel{r}{r}}$ & 导点点 \\
\hline & $\infty$ & 至 & हो : & 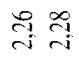 & if & & $\infty$ & $\stackrel{?}{g}$ & $\stackrel{1}{2} \stackrel{g}{8}$ & 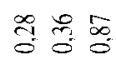 \\
\hline & $\stackrel{\infty}{z}$ & के 8 & 声 & $\begin{array}{l}2 \\
20\end{array}$ & 点蒠 $\frac{a}{8}$ & & $\stackrel{\infty}{z}$ & $\stackrel{8}{9}$ & : & 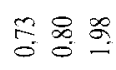 \\
\hline Es & j & 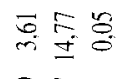 & 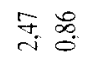 & $\begin{array}{l}a \\
\text { gi } \\
g\end{array}$ & $\begin{array}{l}\cong \\
\text { 월 }\end{array}$ & E & $\Xi$ & ț & वे ृु & $\vec{r} \vec{r}$ \\
\hline$\hat{\Sigma}$ & $\star$ & $\begin{array}{l}g \\
\text { gi } \\
\infty \\
\infty\end{array}$ & $\underset{\sigma}{*}$ & $\begin{array}{l}n \\
2 \\
0 \\
0\end{array}$ & $\begin{array}{l}\bar{\sigma} \approx \\
\infty \\
\infty\end{array}$ & $\stackrel{1}{\Sigma}$ & 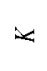 & $\Xi$ & $\underset{\infty}{\stackrel{0}{0}} \underset{-i}{-i}$ & 윽 요용 \\
\hline & 2 & $\begin{array}{l}\bar{\infty} \\
i\end{array}$ & $\overline{3}$ & 官 & $\stackrel{\infty}{\circ} \vec{\infty}$ & & 2 & $\frac{\infty}{0}$ & $\frac{\infty}{6} 8$ & $\overline{~ ন ~}$ \\
\hline & $z$ & 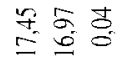 & 导学 & $\begin{array}{l}\text { S寻 } \\
\text { 号 }\end{array}$ & 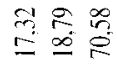 & & $z$ & $\stackrel{\infty}{-}$ & $\stackrel{8}{0} \stackrel{0}{-}$ & g $g$ \\
\hline & $\sim$ & $\stackrel{2}{=} \stackrel{8}{\circ}$ & 용 & 昌 & क⿺ 一 & & $\omega$ & 3 & $\frac{0}{6}$ & ठ용 \\
\hline & $\sum$ & 웜용 & $\stackrel{8}{8} \stackrel{0}{0}$ & $\stackrel{2}{m}$ & 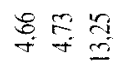 & & $\stackrel{\infty}{\Sigma}$ & گ્ & 导守 & की \\
\hline & $\Xi$ & $\begin{array}{l}\infty \\
\text { in } \\
\text { i d } \\
\text { so }\end{array}$ & $\bar{i}_{i}$ & $\begin{array}{ll}a \\
\infty\end{array}$ & $\stackrel{g}{g} \frac{a}{2} \frac{\sigma}{9}$ & & $\Xi$ & 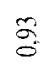 & 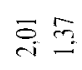 & $\ddot{m} \bar{\sigma}$ \\
\hline$\Sigma$ & 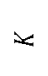 & 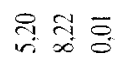 & 寺念 & $\bar{\Xi}$ & $\begin{array}{l}\bar{\sigma}: \bar{z} \\
\text { i }\end{array}$ & $\bar{\alpha}$ & $x$ & $\bar{s}$ & 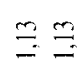 & 导吉要 \\
\hline & 2 & $\Xi \mathscr{O} \stackrel{g}{\sigma}$ & $\frac{0}{0}$ & $8=$ & $\because$ वे & & 2 & $\stackrel{ }{6}$ & $=\frac{9}{0}$ & $\frac{ \pm}{0} \frac{ \pm}{0}$ \\
\hline & $z$ & $\begin{array}{l}0 \\
= \\
=\end{array}$ & $\sqrt{-i}$ & 导是 & 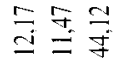 & & $z$ & 8 & $\stackrel{\varrho}{=}$ & 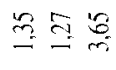 \\
\hline & $\infty$ & 冓㔯 & $\approx 8$ & $\frac{1}{0}$ & 흡 g & & $n$ & $\stackrel{\mathscr{S}}{=}$ & $\stackrel{8}{8}$ & $\frac{9}{6} \frac{1}{6} 8$ \\
\hline & $\stackrel{\infty}{\Sigma}$ & $\stackrel{2}{8}=8$ & S. & $\stackrel{2}{2}$ & $\begin{array}{ll}0 & 0 \\
-i & 0\end{array}$ & & $\stackrel{s c}{\Sigma}$ & $\frac{9}{8}$ & $\frac{2}{0} \stackrel{8}{0}$ & ¿े \\
\hline$\Xi$ & $\Xi$ & $\Rightarrow \stackrel{\infty}{=}$ & $\underset{i}{\mp}$ & $\begin{array}{l}2 \\
\infty \\
\infty\end{array}$ & $\begin{array}{l}\infty \\
\stackrel{D}{\Xi}\end{array}$ & $\cong$ & $\Xi$ & वे & $\stackrel{8}{8}$ & 용 \\
\hline 言 & $\infty$ & 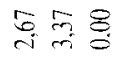 & $\stackrel{\circ}{\circ}$ & 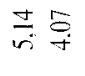 & 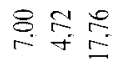 & 佘 & $\infty$ & $\frac{\pi}{8}$ & 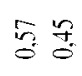 & $\underset{8}{0}$ \\
\hline & 2 & 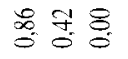 & $\frac{D}{6}$ & 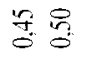 & $g 8$ & & 2 & 8 & $\stackrel{8}{8}$ & $\stackrel{g}{g} \Xi \vec{g}$ \\
\hline & $z$ & $\begin{array}{l} \pm \\
\dot{\sigma} \\
\dot{\sigma}\end{array}$ & 告 & $3 \underset{+}{9}$ & 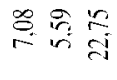 & & $z$ & 昂. & $\sqrt{5} 8$ & 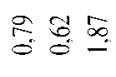 \\
\hline & & 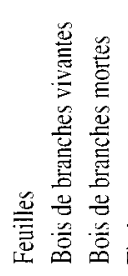 & 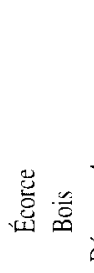 & & 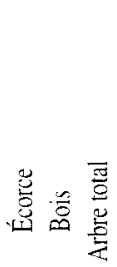 & & & 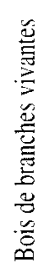 & 递 & 总芯 \\
\hline
\end{tabular}


la variabilité observée n'est pas liée aux traitements. L'explication de la variance est toujours suffisante pour autoriser leur utilisation comme modèles prévisionnels du contenu minéral en fonction de la dimension de l'arbre.

Le contenu minéral des peuplements est en relation avec leur biomasse et augmente avec la fertilisation (tableau X). L'immobilisation moyenne annuelle dans la matière ligneuse aérienne totale varie de 2 à $8 \mathrm{~kg}$ pour $\mathrm{N}, 0,2$ à $0,8 \mathrm{~kg}$ pour $\mathrm{P}, 2$ à $8 \mathrm{~kg}$ pour $\mathrm{K}$, 2,5 à $\mathrm{I} 0$ pour Ca et 0,7 à $3 \mathrm{~kg}$ pour $\mathrm{Mg}$ en fonction des traitements, soit d'un facteur variant de 1 à 4 comme la biomasse.

\section{DISCUSSION}

Les sols sont caractérisés par une discontinuité granulométrique importante conduisant à des phénomènes d'hydromorphie en hiver et à un dessèchement superficiel important en été. L'enracinement est limité aux horizons superficiels ce qui augmente encore les difficultés d'alimentation en eau pendant la saison de végétation. De plus, les horizons profonds dont la richesse minérale est la plus importante, sont peu ou pas explorés par les racines du peuplier. Il peut s'agir : i) soit d'un phénomène mécanique lié à la différence de compacité entre les couches limoneuse et argileuse, classiquement observé dans les pélosolspseudogleys mal structurés, ii) soit d'un phénomène d'asphyxie des racines dans le pseudogley riche en argile (45\%), mal structuré, compact avec une remobilisation du fer importante traduisant les phénomènes temporaires de déficit en oxygène, ou iii) soit évidemment d'une combinaison des deux effets.

Les effets de la fertilisation sont très marqués en valeur relative, mais la production reste faible en valeur absolue, y compris dans le meilleur traitement $(4,8 \mathrm{t}$ de MS $\mathrm{ha}^{-1} \mathrm{an}^{-1}$ de production ligneuse aérienne moyenne à 9 ans). L'interaction entre les traitements et les blocs est mise en évidence par les coefficients de variation calculés pour les divers compartiments de la biomasse (tableau VI). Ils diminuent très nettement avec l'efficacité du traitement (de 60 à 25) indiquant que les différences locales sont très bien compensées par la fertilisation, peut être justement parce que le prélèvement des arbres est limité aux horizons supérieurs.

Il semble que le cumul de conditions telles que :

- la mise en place du taillis dans des conditions non optimales,

- les sécheresses des années suivant l'installation (été 1984 et 1985), et le froid exceptionnel de l'hiver 1985 ,

- une concurrence herbacée insuffisamment maitrisée,

-le fait qu'il s'agisse d'une première rotation avec un système radiculaire encore peu développé,

- le maintien sur pied du taillis pendant 9 ans se traduisant par des productions très faibles pendant les deux dernières années. conduisent à une estimation biaisée du potentiel réel de production dans cette situation.

La production en deuxième rotation devrait apporter une information plus exacte. sans que l'on doive s'attendre à des résultats très élevés. On peut estimer que $7 \mathrm{t}$ de $\mathrm{MS} h \mathrm{a}^{-1} \mathrm{an}^{-1}$ de matière ligneuse aérienne totale devraient représenter un objectif en seconde rotation, si la récolte a lieu à $7 \mathrm{au}$ lieu de 9 ans, compte tenu que les deux dernières saisons de végétation ont conduit à des productions pratiquement nulles.

Un indice global d'efficience des éléments nutritifs pour produire la biomasse peut être calculé par le rapport biomasse produite/élément contenu dans le même compartiment (tableau XI). Cette efficience globale varie peu avec les traitements, mais les traitements fertilisés sont très générale- 
Tableau XI. Efficience des éléments nutritifs's pour produire la biomasse (données en t de biomasse par unité d'élément).

\begin{tabular}{|c|c|c|c|c|c|c|c|c|c|c|}
\hline & $\begin{array}{c}N \\
\text { tronc }\end{array}$ & $\begin{array}{c}P \\
\text { tronc }\end{array}$ & $\begin{array}{c}K \\
\text { tronc }\end{array}$ & $\begin{array}{c}\mathrm{Ca} \\
\text { monc }\end{array}$ & $\begin{array}{c}M_{g} \\
\text { tronc }\end{array}$ & $\begin{array}{c}N \\
\text { ligneur }\end{array}$ & $\begin{array}{c}P \\
\text { ligneux }\end{array}$ & $\begin{array}{c}K \\
\text { ligneut }\end{array}$ & $\begin{array}{c}\text { Ca } \\
\text { ligneux }\end{array}$ & $\begin{array}{c}M_{\mathrm{g}} \\
\text { ligheux }\end{array}$ \\
\hline Témoin & 0,64 & 5,58 & 0,69 & 0,45 & 1,61 & 0,58 & 5,25 & 0,65 & 0,45 & 1,60 \\
\hline P1 & 0,70 & 6,28 & 0,7 & 0,48 & 1,77 & 0,63 & 5,72 & 0,65 & 0,48 & 1,76 \\
\hline $\mathrm{NP} 2 \mathrm{~K} \mathrm{l}$ & 0,72 & 6.71 & 0,67 & 0.49 & 1,87 & 0,62 & 5,99 & 0,61 & 0,49 & 1,85 \\
\hline $\mathrm{NP} 1 \mathrm{~K} 1 \mathrm{Ca}$ & 0,71 & 6,76 & 0,66 & 0,49 & 1,89 & 0,61 & 5.97 & 0,59 & 0,48 & 1,78 \\
\hline TCR Populus a & 0,60 & 3,05 & 0,7 & 0,21 & 3,47 & 0,45 & 2,51 & 0,62 & 0,17 & 3 \\
\hline
\end{tabular}

${ }^{a}$ Moyenne observée sur les TCR de valléc installés par l’Afocel el étudiés par Ranger et Nys ( 1996).

ment les plus efficients (tous les éléments sauf $\mathrm{K}$ ), tendant ainsi à montrer que l'apport de fertilisants n'a pas conduit à une consommation supplémentaire d'éléments nutritifs autre que proportionnelle à l'augmentation de la production. La comparaison avec les TCR de peupliers installés sur des sols plus riches que ceux d'Uxeloup montre que ce TCR est également voire plus efficient que TCR de sols riches pour tous les éléments sauf $\mathrm{Mg}$.

La situation de ce TCR de peuplier dans les relations biomasse produite-consommation d'éléments nutritifs établies pour les taillis extensifs et intensifs (Ranger et Nys, 1996) est singulière. Compte tenu de sa faible production, ce peuplement (quel que soit le traitement), se situe dans le domaine des taillis extensifs et non dans celui des taillis intensifs pour les relations entre biomasse et éléments immobilisés. Il est toutefois impossible de déterminer avec ce seul exemple de TCR peu productif, si ce peuplement entre ou non dans la relation générale obtenus pour les TCR plus productifs (figure 3).

Ce TCR semble toutefois plus efficient pour l'utilisation des éléments nutritifs que les TCR de vallée étudiés précédemment. Il est possible également que la relation production de biomasse-consommation d'élé- ments nutritifs ne suive pas un modèle linéaire comme cela semblait se dessiner à partir de l'échantillon initial des TCR étudiés. Dans ce dernier cas, l'efficience du genre Populus ne serait pas constante mais varierait avec la richesse du milieu.

À titre d'exemple, l'immobilisation moyenne annuelle d'éléments minéraux d'un clone de peuplier de forte production (clone Boleare d'un TCR de l'Afocel installé sur un sol alluvial de l'Aisne de production ligneuse aérienne totale de $14 \mathrm{t}$ de MS ha ${ }^{-1} \mathrm{an}^{-1}$ ) est de $42 \mathrm{~kg}$ de $\mathrm{N}, 7 \mathrm{~kg}$ de $\mathrm{P}$, $35 \mathrm{~kg}$ de $\mathrm{K}, 70 \mathrm{~kg}$ de $\mathrm{Ca}$ et $5,3 \mathrm{~kg}$ de $\mathrm{Mg}$. Celle d'un peuplement de Châtaignier (installé sur un sol brun léssivé en Charente) de production identique au peuplier fertilisé d'Uxeloup (4,7 t de MS ha-1 $\mathrm{an}^{-1}$ ) est de $6,4 \mathrm{~kg}$ de $\mathrm{N}, 0,4 \mathrm{~kg}$ de $\mathrm{P}, 3 \mathrm{~kg}$ de $\mathrm{K}, 7,5 \mathrm{~kg}$ de Ca et $1,8 \mathrm{~kg}$ de $\mathrm{Mg}$. À production équivalente, le peuplier de faible production présente une immobilisation de l'ordre de grandeur de celle du châtaignier.

\section{CONCLUSION}

Le test effectué sur un sol forestier de faible qualité, tant pour sa fertilité minérale que pour son potentiel d'alimentation en eau, montre que la production obtenue en pre- 
(kg par ha)

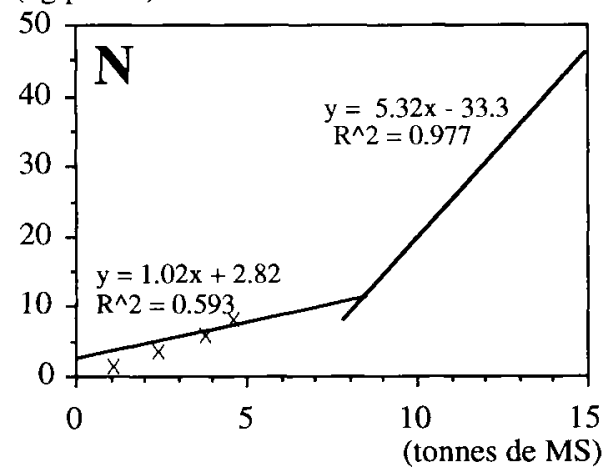

(kg par ha)

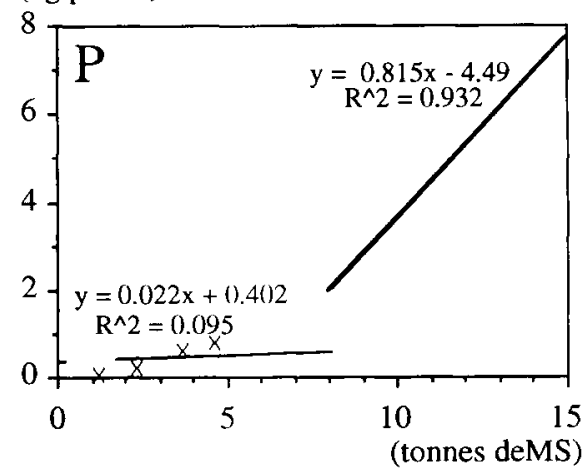

(kg par ha)

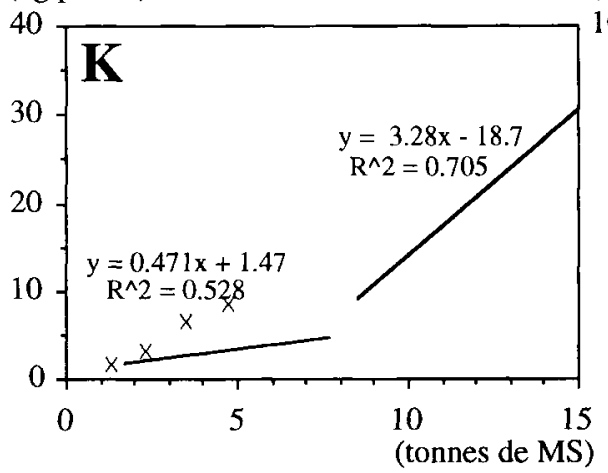

(kg par ha)

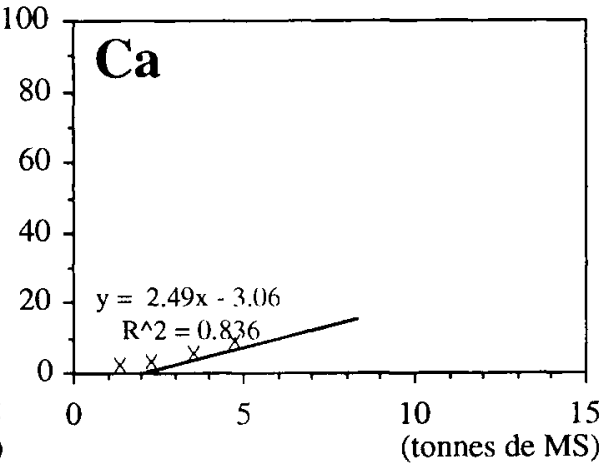

(kg par ha)

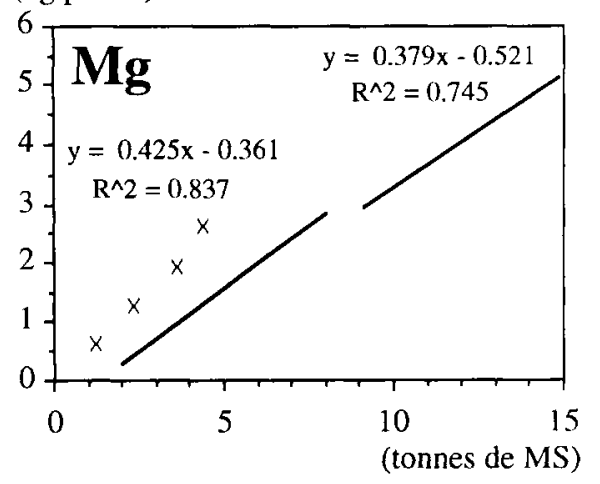

taillis extensifs

taillis intensifs

Fig 3. Position des traitements d'Uxeloup dans la relation générale minéralomasse $=$ f(biomasse ligneuse aérienne totale) des taillis extensifs et intensifs (Ranger et Nys, 1996). 
mière rotation est faible. L'amélioration due à la fertilisation est importante en valeur relative, mais loin d'être suffisante pour un développement industriel puisque la production moyenne sur le meilleur traitement de l'essai atteint à peine $5 \mathrm{t}$ de MS $\mathrm{ha}^{-1} \cdot \mathrm{an}^{-1}$. Il est cependant prématuré de conclure définitivement et le test doit être poursuivi sur une deuxième rotation où une amélioration substantielle devrait être observée. Il est cependant peu probable que les $7 \mathrm{t}$ de production moyenne annuelle puissent être dépassées, ce qui ne devrait toutefois pas conduire à une rentabilité économique de cette production ; l'aspect technique est intéressant à tester pour pouvoir répondre de manière objective à cette interrogation. Une installation plus soignée (sol labouré plus profondément), un meilleur contrôle de la végétation concurrente, permettraient probablement, si on ne se heurte pas à de très mauvaises conditions climatiques en début de cycle, d'atteindre une meilleure production (Bonduelle et Berthelot, 1994b). Ces sols peuvent par ailleurs être valorisés dans des productions forestières plus classiques moins susceptibles aux fluctuations climatiques, en particulier si l'enracinement des peuplements peut $s$ 'installer dans l'horizon argileux mal structuré, où le risque d'asphyxie des racines est important, mais ne produisant qu'à beaucoup plus long terme que le TCR de peuplier.

La consommation d'éléments minéraux par ce peuplement est faible et se traduit par de bons indices d'efficience. L'enrichissement du sol est encore net pour les éléments tels $\mathrm{Ca}$ et $\mathrm{P}$ et indirectement $\mathrm{N}$ et une seconde rotation devrait pouvoir supporter un apport complémentaire de fertilisants ne correspondant qu'à la stricte restitution des exportations ; les rémanents d'exploitation fourniront les éléments au début de la rotation, les fertilisants ne devraient être apportés que lorsque la demande des peuplements sera la plus élevée ( 2 ou $3^{\mathrm{e}}$ année).
Remerciements : Les auteurs remercient l'Ademe pour son soutien financier dans le cadre du contrat programme de recherche Ademe-Inra sur les taillis à courte rotation de peuplier, convention 4.01.0032, l'Office national des forêts pour son aide efficace lors des diverses phases de cette expérimentation, ainsi que la Caisse de retraite des caisses d'épargne, propriétaire du terrain.

\section{RÉFÉRENCES}

Afocel (1982) Culture de biomasse ligneuse. Taillis à courte révolution. Publication Afocel, domaine de l'Étançon, 213 p

Anne P (1945) Sur le dosage rapide du carbone organique des sols. Ann Agro 2, 161-172

Bonduelle P (1989) Sylviculture du taillis en taillis à courtes rotations (TCR). Afocel Nord-Ouest, 49800 Trélazé, $41 \mathrm{p}$

Bonduelle P, Berthelot A (1994a) La culture de TCR de peuplier. I. Objectits et choix des sols. Fiches Informations-forêt $2,482,145-156$

Bonduelle P, Berthelot A (1994b) La culture de TCR de peuplier. II. Itinéraire technique. Fiches Informations-forêt 2, 482, 157-172

Bonneau M (1994) Résultats de l'essai de fertilisation d'un taillis à courte révolution de peuplier Béaupré en forêt de Luthenay-Uxeloup (Nièvre). Document Interne Inra centre de Nancy, $15 \mathrm{p}$

Bonneau M (1995) Fertilisation des forêts dans les pays tempérés. Théoric, bases du diagnostic, conseils pratiques, réalisations expérimentales. Engref, $367 \mathrm{p}$

Carlisle A, Methven IR (1979) The environmental consequences of intensive forestry and the removals of whole trees. In: Proceeding of Biological and Sociological Basis for a Rational Use of Forest Resources for Energy and Organics, MAB congress, Michigan State University, May 1979. $108-120$

Christersson L, Sennerby-Forsse L (1995) Willow and poplar plantation research and plantations in Sweden to-day. Swedish Univ. of Agricultural Sciences, Dept of Ecology and Envir Res Section of Short Rotation Forestry, Report 53, $17 \mathrm{p}$

Duchaufour P, Bonneau M (1959) Une méthode nouvelle du dossage du phosphore assimilable dans les sols forestiers. Bull. Afes 4, 193-198

Deb BC (1960) The estimation of free iron oxydes in soils and clays and their removal. $J$ Soil Sci 1, 212220

Mehra OP, Jackson ML (1960) Iron oxyde removal from soils and clays by a dithionite-citrate system 
buffered with sodium bicarbonate. Clays and Clay minerals 7, 317-327

Miegroet van H, Norby RJ, Tschaplinski TJ (1994) Nitrogen fertilization strategies in a short-rotation sycamore plantation. For Ecol Manag 64, 1.3-24

Pertu K (ed) (1984) Ecology and management of forest biomass production systems. Dept Ecol Environ Res, Swedish University of Agricultural Sciences Report 15,607p

Ranger J, Nys C (1996) Biomass and nutrient content of extensively and intensively managed coppice stands. Forestry 69, 2, 83-102
Rouiller J, Guillet B, Bruckert S (1980) Cations acides échangeables et acidités de surface, approche analytique et incidences pédologiques. $S_{c} i d u$ Sol Ales 2. $161-175$

Shelton MG, Switzer (iL, Nelson LE, Baker JB, Mueller CW (1982) The development of cottonwood plantations on alluvial soils: dimensions, volume, phytomass, nutrient content and other characterislics. Agro For Exp Stat Techn Bull 113,43 p

Steinbeck K. May JT (1971) Productivity of very young Platamus occidentalis L plantings grown at various spacings. Proccedings Iufro Congress, Gainsville, FL, États-Unis, 153-162 